\title{
Long-term overexpression of glucokinase in the liver of transgenic mice leads to insulin resistance
}

\author{
T. Ferre, E. Riu, S. Franckhauser, J. Agudo, F. Bosch \\ Department of Biochemistry and Molecular Biology, School of Veterinary Medicine and Center of Animal Biotechnology \\ and Gene Therapy, Universitat Autònoma de Barcelona, Bellaterra, Spain
}

\begin{abstract}
Aims/hypothesis. Glucokinase overexpression in the liver increases glucose uptake and utilization, and improves glucose tolerance in young transgenic mice. Here, we examined the long-term effects of hepatic overexpression of glucokinase on glucose homeostasis. Moreover, we determined whether glucokinase overexpression counteracted high-fat diet-induced insulin resistance.

Methods. Transgenic mice overexpressing glucokinase in liver under the control of the phosphoenolpyruvate carboxykinase promoter, fed either a standard diet or a high-fat diet, were studied. We used non-transgenic littermates as controls.

Results. Transgenic mice over 6 months old developed impaired glucose tolerance. In addition, at 12 months of
\end{abstract}

age, transgenic mice showed mild hyperglycaemia, hyperinsulinaemia and hypertriglyceridaemia. In spite of increased glucokinase activity, the liver of these mice accumulated less glycogen and increased triglyceride deposition. When 2-month-old glucose-tolerant mice were fed a high-fat diet, transgenic mice gained more body weight and became hyperglycaemic and hyperinsulinaemic. This was concomitant to glucose intolerance, liver steatosis and whole-body insulin resistance. Conclusion/interpretation. Long-term overexpression of glucokinase increases hepatic lipogenesis and circulating lipids, which lead to insulin resistance. Our results also suggest that the liver plays a key role in the onset of diabetes. [Diabetologia (2003) 46:1662-1668]

Keywords Transgenic mice, glucokinase, high-fat diet, glucose metabolism, insulin resistance, Type 2 diabetes.
Glucokinase (GK) is the main glucose-phosphorylating enzyme in the liver and pancreatic beta cells. Glucose transport and phosphorylation are the first steps in glucose utilization in the liver, where GK contributes to glucose disposal. Several studies in vitro have shown that GK activation is needed for glycolysis and glycogen synthesis $[1,2,3,4]$. Similarly, in transgenic

Received: 17 June 2003 / Revised: 28 July 2003

Published online: 12 November 2003

(C) Springer-Verlag 2003

Corresponding author: F. Bosch, Department of Biochemistry and Molecular Biology, School of Veterinary Medicine and Center of Animal Biotechnology and Gene Therapy, Universitat Autònoma de Barcelona, 08193 Bellaterra, Spain

E-mail: fatima.bosch@uab.es

Abbreviations: GK, glucokinase; PEPCK, phosphoenolpyruvate carboxykinase. mice overexpressing GK in the liver, intracellular glucose 6-phosphate, glycogen and L-pyruvate kinase activity are increased, indicating that the activation of GK can induce glycolysis and glycogen synthesis in vivo [5]. These transgenic mice have reduced glycaemia, insulinaemia and blood glucose after an intraperitoneal glucose tolerance test, indicating that GK overexpression increased blood glucose disposal by the liver [5]. Similar results have also been obtained in transgenic mice expressing the human GK gene in the liver [6] and in mice overexpressing one or more extra copies of the entire mouse GK gene locus [7].

Since the decrease in glucokinase gene expression in diabetic animals could contribute to hyperglycaemia, the effect of hepatic GK overexpression has been studied in animal models of Type 1 and Type 2 diabetes. Thus, streptozotocin-treated GK-expressing transgenic mice maintained normal levels of GK activity, 
which were associated with increased glycolysis and reduced gluconeogenesis. This leads to normalization of blood glucose in the absence of insulin [8]. Furthermore, a high-fat diet did not induce diabetes in transgenic mice overexpressing one copy of the GK gene [9]. In these mice, hepatic GK overexpression reduced hyperglycaemia and hyperinsulinaemia induced by a high-fat diet. Moreover, adenovirus-mediated expression of GK in the liver improves glucose tolerance and reduces hyperinsulinaemia in high-fat dietinduced diabetes in mice [10]. These results point to overexpression of GK as a possible gene therapybased approach to reduce hyperglycaemia in diabetes.

However, high levels of adenovirus-mediated GK overexpression decrease blood glucose concentrations with concomitant increases in circulating free fatty acids and triglycerides [11]. Moreover, an increase in GK activity has been described in obese-diabetic subjects [12] and in obese hyperinsulinaemic $(f a / f a)$ Zucker rats [13], suggesting that hepatic GK overexpression could have diabetogenic effects. Therefore, the aim of this study was to examine the long-term effects of GK overexpression in the liver of transgenic mice expressing a $P E P C K / G K$ chimeric gene. We found that old transgenic mice with increased GK activity developed glucose intolerance and hepatic lipid accumulation. In addition, these mice were more sensitive to high-fat diet-induced diabetes. These results suggest that GK overexpression might exacerbate diabetes and confirm the key role of the liver in its onset.

\section{Materials and methods}

Treatment of mice. Transgenic mice expressing the GK gene in liver under the control of the PEPCK promoter in a C57B16;SJL mixed genetic background were used [5]. The line of transgenic mice with the highest copy number and activity were used in this study [5]. We used littermates as controls for the transgenic animals. Mice were fed ad libitum with a standard diet (Panlab, Barcelona, Spain) and maintained under a light-dark cycle of $12 \mathrm{~h}$ (lights on at 8:00 a.m.). For the experimental induction of Type 2 diabetes, mice were kept in individual cages and fed a high-fat diet (TD 88137, Harlan Teklad Laboratory, Madison, Wis., USA) for up to 12 weeks. Where stated, mice were starved for $16 \mathrm{~h}$. Animals were anaesthetized with isofluorane (between 9 and 11 a.m.), killed by decapitation and blood and tissue samples obtained. In the experiments described below, heterozygous male mice aged 8 weeks at the start of the diet were used. All experimental procedures involving mice were approved by the Ethics and Experimental Animal Committee of the Universitat Autònoma de Barcelona.

Enzyme, metabolite and hormone assays. To measure GK activity, liver samples were homogenized in an ice-cold buffer (pH 7.4) containing $50 \mathrm{mmol} / \mathrm{l}$ Tris- $\mathrm{HCl}, 300 \mathrm{mmol} / \mathrm{l}$ sucrose, $100 \mathrm{mmol} / \mathrm{l} \mathrm{KCl}, 1 \mathrm{mmol} / \mathrm{l}$ ethylenediamine tetraacetic acid, and $0.7 \mu \mathrm{l} / \mathrm{ml} \beta$-mercaptoethanol. GK activity was analyzed in $12000 \times g$ supernatants [14] and was calculated as the difference between the glucose phosphorylation capacity at $100 \mathrm{mmol} / \mathrm{l}$ and $0.5 \mathrm{mmol} / \mathrm{l}$ glucose. To measure the concentration of metabolites, liver samples were immediately frozen in liquid $\mathrm{N}_{2}$. The concentrations of hepatic glycogen, and glucose 6-phosphate were measured in perchloric extracts, which were adjusted to $\mathrm{pH} 5$ with $5 \mathrm{M} \mathrm{K}_{2} \mathrm{CO}_{3}$ for glycogen, and to $\mathrm{pH} 7$ for glucose 6-phosphate. Glycogen levels were measured by the $\alpha$-amyloglucosidase method [15], and glucose 6-phosphate was determined enzymatically [16]. Hepatic triglyceride content was determined by extracting total lipids from liver samples with chloroform-methanol $(2: 1 \mathrm{vol} / \mathrm{vol})$ as described by another study [17] separating the chloroform and methanolwater phases. Triglycerides were then quantified spectrophotometrically using an enzymatic assay kit (GPO-PAP, Roche Molecular Biochemicals, Mannheim, Germany). Glucose was measured enzymatically (Glucoquant ${ }^{\circledR}$, Roche) in serum. Glucose was also determined in $5 \mu$ of blood by using Glucometer $^{\circledR}$ Elite analyzer (Bayer Diagnostics Europe, Dublin, Ireland). Serum triglycerides were determined enzymatically (GPO-PAP, Roche, Germany). Serum insulin concentrations were measured by radioimmunoassay (CIS, Biointernational, Gif-Sur-Yvette, France), using rat insulin (Eli Lilly, Indianapolis, Ind., USA) as standard.

Glucose and insulin tolerance tests. For glucose tolerance test, awake control and transgenic mice fasted overnight $(16 \mathrm{~h})$ with free access to water were given an intraperitoneal injection of glucose ( $1 \mathrm{~g} / \mathrm{kg}$ of body weight). Blood samples were obtained from the tail vein before the glucose injection and at the indicated time points after the glucose load, and glucose concentration was measured. For insulin tolerance test, insulin (0.75 IU/kg body weight; Humulin regular, Eli Lilly) was injected intraperitoneally into awake, fed control and transgenic mice. Glucose concentration was determined in blood samples obtained from the tail vein at the indicated time points after the insulin injection.

Histological analysis. The livers from control and transgenic mice were fixed for 12 to $24 \mathrm{~h}$ in formalin, embedded in paraffin and sectioned. Sections were stained with haematoxylin/ eosin.

Statistical Analysis. Enzyme activities, serum parameters and metabolite concentrations are expressed as the means \pm SEM. Analysis of statistical differences between data were carried out using the Student-Newmann-Keuls test. A $p$ value of less than 0.05 was considered statistically significant.

\section{Results}

Long-term overexpression of $G K$ in the liver led to insulin resistance. To examine whether long-term overexpression of GK affected glucose disposal, an intraperitoneal glucose tolerance test was carried out in transgenic mice at different ages (Fig. 1). In contrast to 2-month-old transgenic mice, which showed increased glucose disposal, 3-month-old transgenic animals showed no improvement in glucose tolerance compared with control animals (Fig. 1A). Furthermore, 6-month-old transgenic mice reached higher blood glucose concentrations than control mice and did not recover basal glucose at 180 min after glucose administration (Fig. 1B). Similar results were observed in 12-month-old transgenic mice (data not shown). This indicates that GK overexpression leads to progressive, age-dependent glucose intolerance. 
3 months

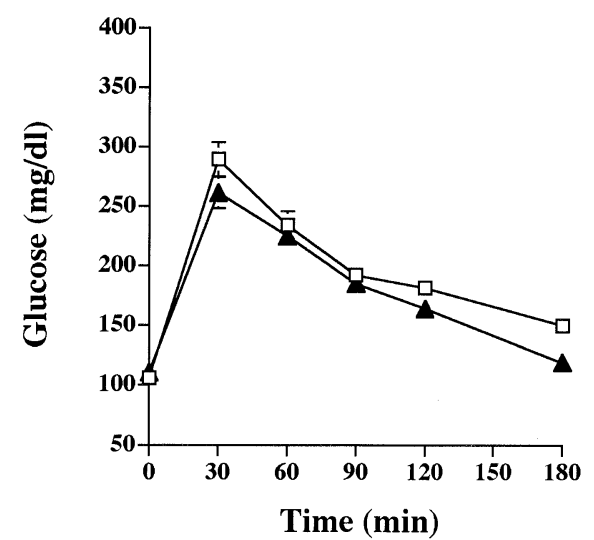

Fig. 1. Intraperitoneal glucose tolerance test. Overnight-starved transgenic $(\boldsymbol{\Delta})$ and control $(\square)$ (3- and 6-month-old) mice were given an i.p. injection of $1 \mathrm{mg}$ of glucose per $\mathrm{g}$ body weight.
6 months

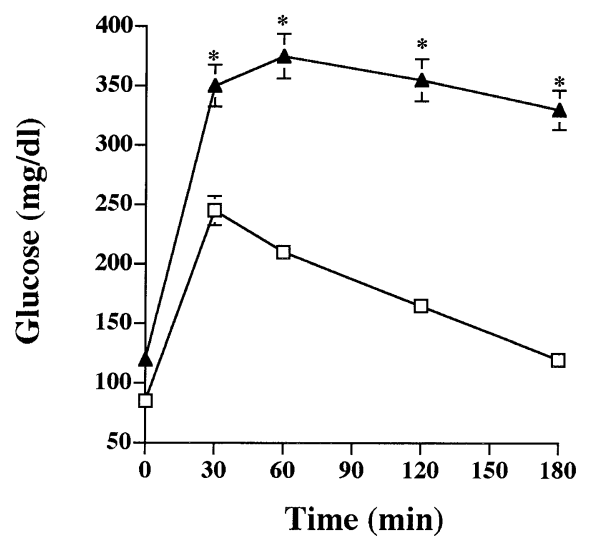

Blood samples were taken from the tail vein of the same animals at the times indicated. Results are means \pm SEM of 8 transgenic and 10 control mice. $* p<0.05$ vs. control mice

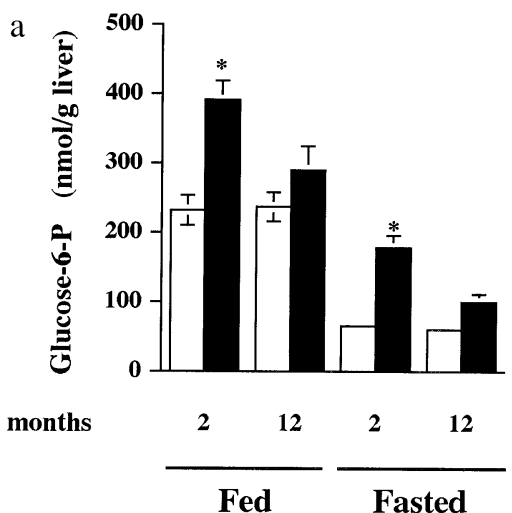

Fig. 2a-c. Glucose-6-P, glycogen and triglyceride content in the liver of transgenic mice expressing glucokinase. The concentration of a: glucose 6-phosphate, $\mathbf{b}$ : glycogen and $\mathbf{c}$ : triglyceride was measured in liver extracts obtained from fed and fasted control and transgenic mice. Results are means \pm SEM of 18 different animals in each group. $* p<0.05$ vs. control mice

Furthermore, in contrast to 2-month-old fasted transgenic mice, 12-month-old mice presented mild hyperglycaemia, hyperinsulinaemia (about two-fold increase) and an increase in triglyceride levels (about 1.6-fold) compared with control mice (Table 1). These findings suggested that transgenic mice developed insulin resistance with aging. Since GK was under the control of the PEPCK promoter, GK activity was determined in fasted mice. 2-month-old transgenic mice showed higher (about five-fold) GK activity than controls (Table 2). However, 12-month-old animals showed only a two- fold increase in GK activity. Thus, GK activity decreased in transgenic mice when older.

2-month-old transgenic mice accumulate more glycogen and glucose-6-P in the liver than controls both in fed and fasted conditions (Fig. 2A,B). 12-monthold fed control and transgenic mice showed similar

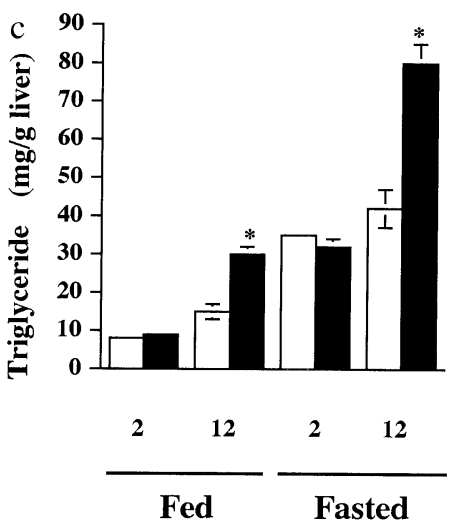

Table 1. Serum parameters in fasted transgenic mice overexpressing GK

\begin{tabular}{lcll}
\hline & $\begin{array}{l}\text { Glucose } \\
(\mathrm{mg} / \mathrm{dl})\end{array}$ & $\begin{array}{l}\text { Insulin } \\
(\mathrm{ng} / \mathrm{ml})\end{array}$ & $\begin{array}{l}\text { Triglycerides } \\
(\mathrm{mg} / \mathrm{dl})\end{array}$ \\
\hline Control & $88 \pm 3$ & $1.5 \pm 0.2$ & $120 \pm 2$ \\
Transgenic & $132 \pm 4 *$ & $3.3 \pm 0.7 *$ & $203 \pm 2 *$ \\
\hline
\end{tabular}

Glucose, insulin and triglycerides in serum of control and transgenic mice were determined. Results are \pm SEM of at least 15 different animals in each group. $* p<0.05$ vs. control mice

Table 2. Liver glucokinase activity

\begin{tabular}{lcc}
\hline & \multicolumn{2}{c}{ Glucokinase (mU/mg prot) } \\
\cline { 2 - 3 } & 2 months & 12 months \\
\hline Control & $4 \pm 2$ & $5 \pm 3$ \\
Transgenic & $19 \pm 2 *$ & $10 \pm 3^{*}$ \\
\hline
\end{tabular}

Glucokinase activity was measured in liver extracts of fasted control and transgenic mice. Results are the means \pm SEM of 12 transgenic mice from each line and 10 control mice. $* p<0.05$ vs. control mice 
concentrations of glucose-6-P. However, fasted transgenic mice showed an increase in glucose-6-P that was parallel with the increase in GK activity (Fig. 2A). In spite of similar concentrations of glucose-6-P in transgenic and control mice, 12-month-old fed transgenic mice showed a marked decrease (about $70 \%$ ) in hepatic glycogen content (Fig. 2B). Upon fasting, glycogen storage in both control and transgenic animals was nearly completely mobilized. To determine whether the decrease in glycogen storage was parallel to increased lipid metabolism, intrahepatic triglyceride content was measured in old animals. In fasted and fed conditions, hepatic triglyceride concentration was higher in transgenic mice (about 1.5 and two-fold respectively) than in controls (Fig. 2C). All these results suggested that transgenic mice showed altered hepatic glucose and lipid metabolism.

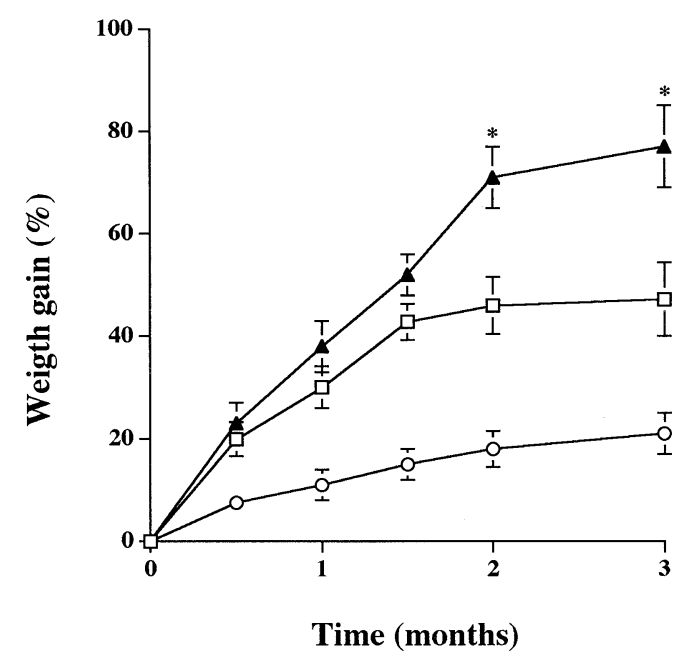

Fig. 3. Effect of a high-fat diet in body weight gain of transgenic mice overexpressing GK in liver. Changes in body weight of control mice fed a standard diet $(\bigcirc)$, and control $(\square)$ and transgenic $(\mathbf{\Delta})$ mice fed a high-fat diet for 3 months are shown. Data are means \pm SEM of 12 mice for each group. $* p<0.05 \mathrm{Tg}$-fat vs. Con-fat

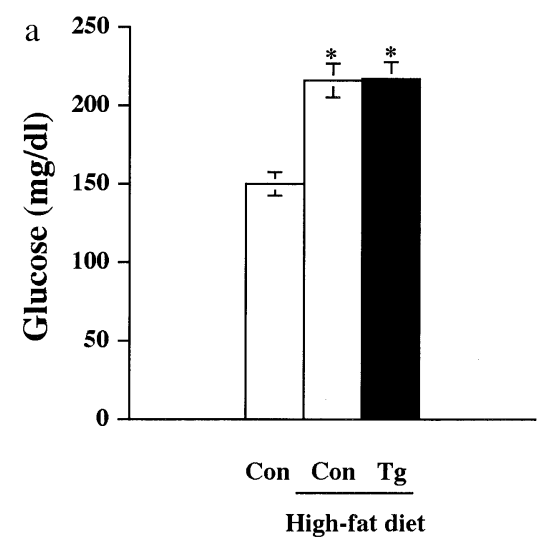

Fig. 4a, b. Serum insulin and blood glucose concentrations. a: Glucose and $\mathbf{b}$ : Insulin were measured in control mice fed a standard diet and in control and transgenic mice after 3 months
Transgenic mice overexpressing GK were not protected from diet-induced obesity and insulin resistance. After 3 months on a high-fat diet, transgenic mice had gained about $80 \%$ body weight whereas control mice only gained $40 \%$ (Fig. 3). In contrast, control mice fed a standard diet showed an increase of about $15 \%$ in body weight (Fig. 3). Although transgenic mice had increased body weight, food intake was similar to that of controls $(3.5 \pm 0.1$ and $3.3 \pm 0.1 \mathrm{~g} / \mathrm{day}$ for control and $\mathrm{Tg}$ mice). Thus, overexpression of GK did not protect mice from diet-induced obesity. After the feeding of a high-fat diet, control mice were mildly hyperglycaemic and hyperinsulinaemic (about three-fold increase) compared to controls fed a standard diet. Transgenic mice showed a similar increase in glycaemia to controls (Fig. 4A). However, transgenic mice had an increase in serum insulin levels (about six-fold compared to control mice fed a standard diet and about two-fold compared to control mice fed a highfat diet) (Fig. 4B). In addition, when an intraperitoneal glucose tolerance test was carried out after 8 weeks in a high-fat diet, transgenic mice reached higher blood glucose concentrations than controls and did not recover basal glucose at $180 \mathrm{~min}$. Moreover, blood glucose concentrations in control mice were higher than those of control mice fed a standard diet (Fig. 5A).

Whole-body insulin sensitivity of transgenic mice was measured after 2.5 month on a high-fat diet by an insulin tolerance test. In fat-fed transgenic mice, the hypoglycaemic effect of insulin was abolished while fat-fed control mice showed a reduced response to insulin compared to control mice fed a standard diet (Fig. 5B). This indicated that transgenic mice were more insulin resistant than controls when fed a highfat diet. Histological analysis of liver sections from mice fed a high-fat diet for 3 months showed dramatic vacuolization due to fat deposition (Fig. 6). Although control mice showed mild lipid accumulation, transgenic mice developed liver steatosis. All these results indicate that transgenic animals under a high-fat diet showed an exacerbated diabetic phenotype.

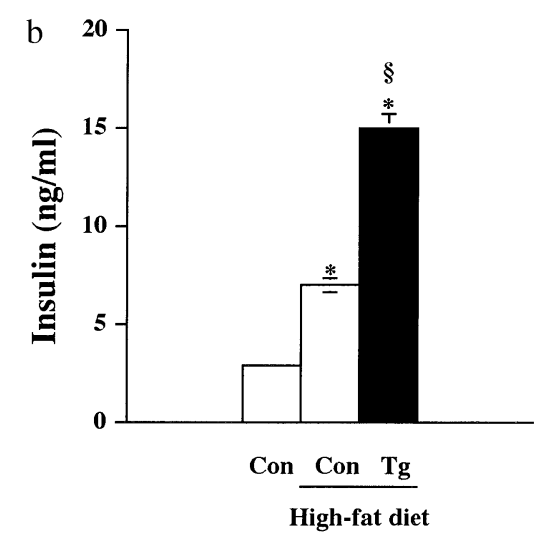

on a high-fat diet in fed conditions. Results are means \pm SEM of 12 animals for each group. ${ }^{*} p<0.05$ vs. control mice; $\S p<0.05$ Tg-fat vs. Con-fat 


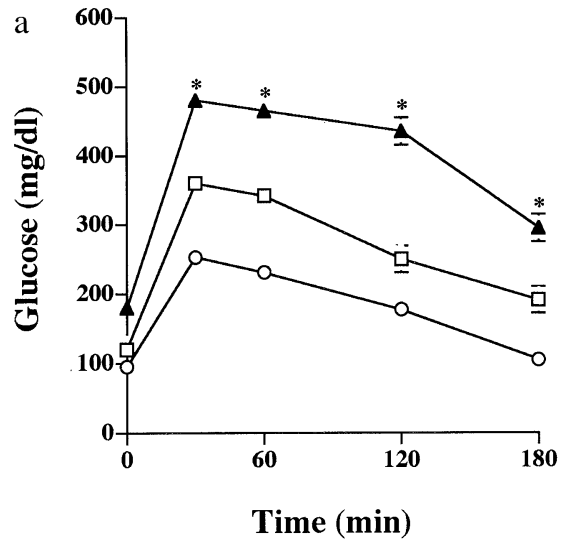

Fig. 5a, b. Intraperitoneal glucose and insulin tolerance tests. a: Intraperitoneal glucose tolerance test. Awake overnightstarved control fed a standard diet $(\bigcirc)$ and control $(\square)$ and transgenic $(\mathbf{\Delta})$ mice after 2.5 months on a high-fat diet were given an intraperitoneal injection of glucose $(1 \mathrm{mg} / \mathrm{g}$ of body weight). Blood samples were taken at the times indicated from the tail vein of the same animal. Results are means \pm SEM of 12 animals for each group. ${ }^{*} p<0.05$; Tg-fat vs. Con-fat. b: Intra-

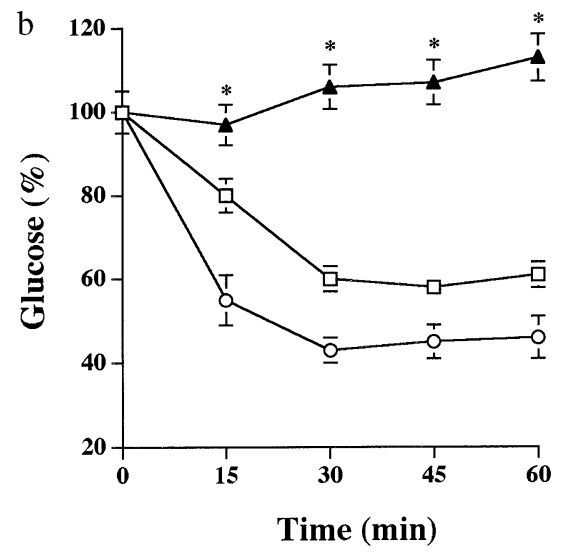

peritoneal insulin tolerance test. Insulin was injected intraperitoneally into awake control fed a standard diet $(\bigcirc)$, and control $(\square)$ and transgenic $(\mathbf{\Delta})$ fed a high-fat diet. Blood samples were taken from the tail vein of the same animals at the time indicated and glucose levels were determined. Results were calculated as percentage of blood glucose values at time 0 for each mouse and are means \pm SEM of six animals for each group. $* p<0.05 ; \mathrm{Tg}$-fat vs. Con-fat

\section{Con}
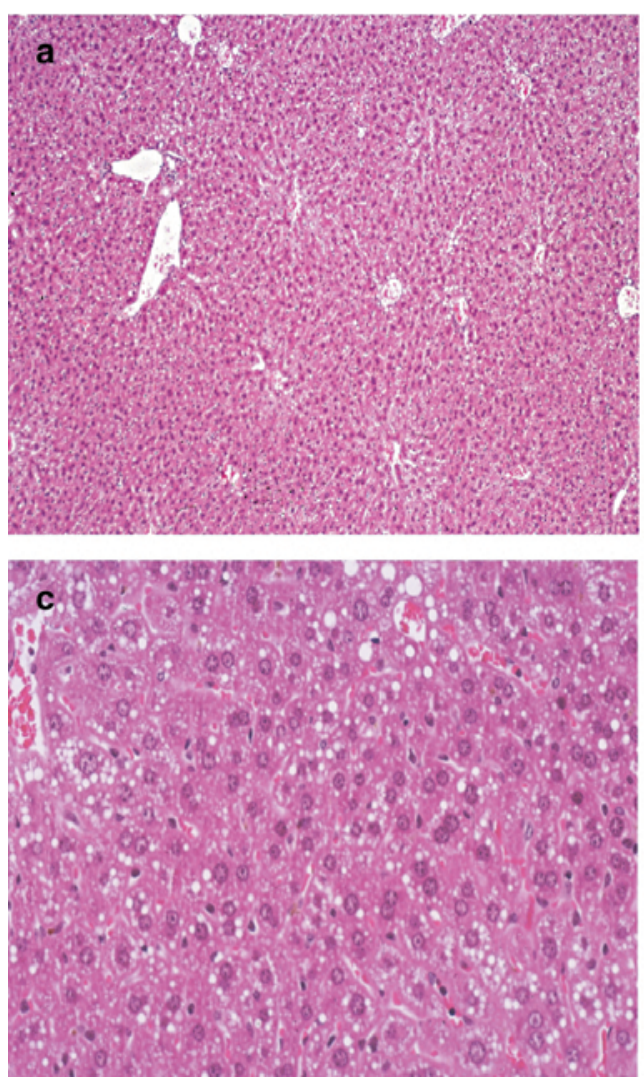

Tg
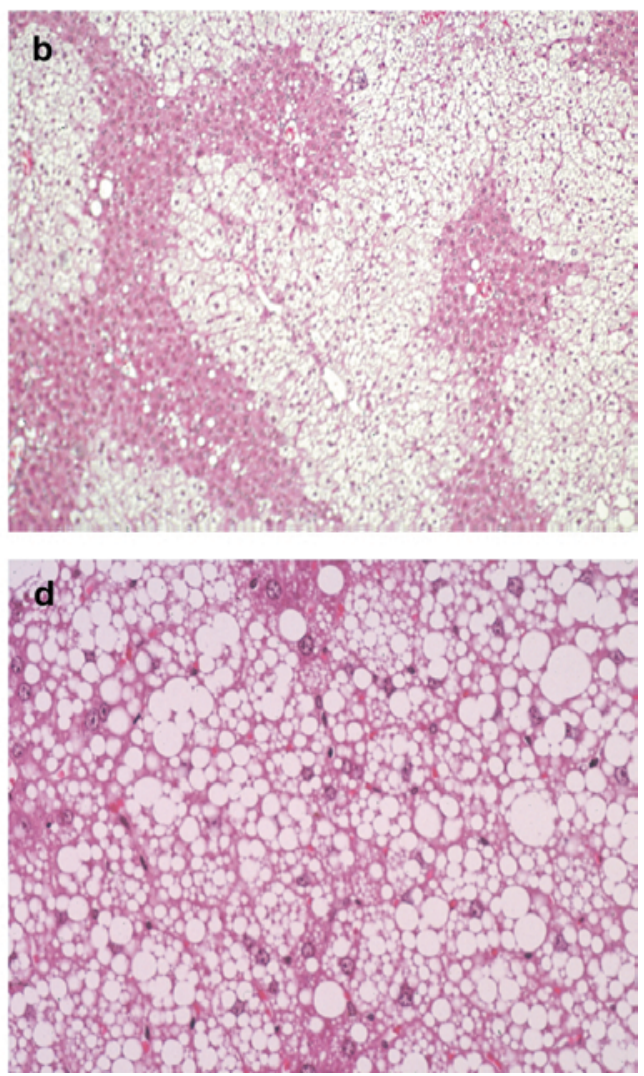

Fig. 6a-d. Long-term overexpression of GK leads to fatty liver. Representative sections of the liver from control (a, c) and transgenic $\operatorname{Tg}(\mathbf{b}, \mathbf{d})$ mice stained with haematoxylin and eosin are presented. (Upper panel $\times 100$ and bottom panel $\times 400$ ) 


\section{Discussion}

Short-term increase in hepatic GK expression increases glycolytic flux and glycogen storage $[1,5,6,7]$. A previous report has shown that a short-term adenovirus-mediated six-fold-increase in GK activity lowers blood glucose concentrations at the cost of an increase in circulating triglyceride and FFA levels [3]. In contrast, a slight increase in GK activity (three-fold) had no effect on glycaemia or serum triglyceride levels [3]. Here we showed that long-term GK overexpression leads to hyperglycaemia, hyperinsulinaemia, hepatic steatosis and increased serum triglyceride levels, resulting in glucose intolerance and lower insulin sensitivity. Thus, our results together with these other findings indicate that GK overexpression has deleterious effects depending on the level and the length of the increase in GK activity.

Transgenic mice overexpressing GK fed a high-fat diet gained more body weight than controls and became glucose intolerant and insulin resistant. In contrast, overexpression of one copy of the GK gene slightly lowers hyperglycaemia and hyperinsulinaemia induced by a high-fat diet [9]. However, these mice also overexpressed GK in beta cells [18] whereas in the present study, transgenic mice specifically overexpressed GK in the liver. Thus, differences between the two transgenic models could be due to the level of hepatic transgene expression and the tissue-specificity.

Type 2 diabetes is characterized by glucose metabolism alterations such as failure of insulin to inhibit hepatic gluconeogenesis and impaired skeletal muscle glucose uptake [19]. However, lipid metabolism is also altered. This is often reflected by increased circulating free fatty acids and triglycerides together with increased fat accumulation in non-adipose tissues [20, 21]. Thus, changes in the equilibrium between glucose and fatty acid metabolism in liver and muscle could be responsible for glucose homeostasis alterations. It has been shown that an increase in glucose metabolism leads to higher hepatic malonyl-CoA levels, which in turn inhibit carnitine palmitoyl transferase 1 (CPT1) activity, resulting in a decrease of long chain fatty acyl $\mathrm{CoA}$ transport into the mitochondria and a decrease of fatty acid oxidation [22, 23]. Consequently, the fat content of the liver and VLDL secretion increase, thus contributing to the development of hypertriglyceridaemia. This, in turn, could lead to triglyceride accumulation in skeletal muscle which might contribute to peripheral insulin resistance. In agreement with this, GK overexpression leads to an increase of fat deposition in the liver and circulating triglyceride levels in transgenic mice. This probably occurred through an increase of fat synthesis from glucose and/or a decrease of fatty acid oxidation due to CPT1 inhibition. The resulting long-chain fatty acyl CoA cytosolic accumulation could inhibit glucose metabolism, thus contributing to hyperglycaemia ob- served in the transgenic animals. Moreover, the increased fat deposition probably decreases hepatic insulin extraction, contributing to hyperinsulinaemia in our transgenic mice [24]. The decrease in glycogen content observed in these mice could be due to hepatic insulin resistance. This is consistent with the negative correlation observed between hepatic glycogen and triglyceride content in rats fed a high-fat diet [25]. Thus, our results suggest a strong interaction between glucose and lipid metabolism and show that a disruption in the equilibrium between the two pathways might lead to insulin resistance.

In humans, fat accumulation in the liver is associated with hepatic insulin resistance, fasting hyperinsulinaemia and hypertriglyceridaemia [26]. Moreover, adipose tissue deficiency in lipodistrophy is associated with hepatic steatosis, increased circulating lipids and insulin resistance $[27,28]$. Insulin sensitivity can be restored in a mouse lipodistrophic model by fat transplantation, probably by recovering the capacity of adipose tissue to accumulate lipids and reducing hepatic fat [29]. These findings together with our results suggest that increased lipid deposition in the liver could result in hyperglycaemia and impaired glucose tolerance.

All these results indicate that a primary alteration in liver might lead to whole-body metabolic alterations. This has also been shown in insulin receptor-deficient mice. Thus, while skeletal muscle has been postulated to be the major site of insulin resistance, mice lacking insulin receptor in muscle have normal whole-body glucose homeostasis [30]. In contrast, liver-specific insulin receptor-deficient mice showed hyperinsulinaemia, hyperglycaemia, insulin resistance and impaired glucose tolerance [31]. Moreover, phosphoenolpyruvate carboxykinase overexpression in the liver leads to increased chronic hepatic glucose production and then to increased insulin secretion, hyperinsulinaemia, hyperglycaemia and insulin resistance [32, 33, 34, 35]. In agreement with this, our results suggest that alteration in hepatic glucose metabolism could be an initial event in the diabetic process. In summary, this study indicates that long-term overexpression of GK results in increased hepatic triglyceride content and circulating lipid levels, which leads to insulin resistance and Type 2 diabetes. Furthermore, our results suggest that gene therapy approaches based on overexpressing GK in the liver for diabetes treatment should consider the side effects of increased GK activity.

Acknowledgements. We thank M. Moya, C.H. Ros and A. Vilalta for technical assistance. T. Ferré was recipient of predoctoral fellowship from Fondo Investigación Sanitaria., E. Riu was recipient of predoctoral fellowship from Direcció General d'Universitats, Generalitat de Catalunya and J. Agudo was recipient of predoctoral fellowship from Ministerio de Educacion, Cultura y Deporte. This research was supported by grants from the Comisión Interministerial de Ciencia y Tecnología (SAF96-0270 and SAF99-0094) Spain, and from the European Community (QLG1-CT-1999-00674). 


\section{References}

1. Valera A, Bosch F (1994) Glucokinase expression in rat hepatoma cells induces glucose uptake and is rate limiting in glucose utilization. Eur J Biochem 222:533-539

2. Seoane J, Gomez-Foix AM, O’Doherty RM, Gomez-Ara C, Newgard CB, Guinovart JJ (1996) Glucose 6-phosphate produced by glucokinase, but not hexokinase I, promotes the activation of hepatic glycogen synthase. J Biol Chem 271:23756-23760

3. O'Doherty RM, Lehman DL, Seoane J, Gomez-Foix AM, Guinovart JJ, Newgard CB (1996) Differential metabolic effects of adenovirus-mediated glucokinase and hexokinase I overexpression in rat primary hepatocytes. J Biol Chem 271:20524-20530

4. Gomis RR, Ferrer JC, Guinovart JJ (2000) Shared control of hepatic glycogen synthesis by glycogen synthase and glucokinase. Biochem J 351:811-816

5. Ferre T, Riu E, Bosch F, Valera A (1996) Evidence from transgenic mice that glucokinase is rate limiting for glucose utilization in the liver. FASEB J 10:1213-1218

6. Hariharan N, Farrelly D, Hagan D et al. (1997) Expression of human hepatic glucokinase in transgenic mice liver results in decreased glucose levels and reduced body weight. Diabetes 46:11-16

7. Niswender KD, Shiota M, Postic C, Cherrington AD, Magnuson MA (1997) Effects of increased glucokinase gene copy number on glucose homeostasis and hepatic glucose metabolism. J Biol Chem 272:22570-22575

8. Ferre T, Pujol A, Riu E, Bosch F, Valera A (1996) Correction of diabetic alterations by glucokinase. Proc Natl Acad Sci USA 93:7225-7230

9. Shiota M, Postic C, Fujimoto Y et al. (2001) Glucokinase gene locus transgenic mice are resistant to the development of obesity-induced type 2 diabetes. Diabetes 50:622629

10. Desai UJ, Slosberg ED, Boettcher BR et al. (2001) Phenotypic correction of diabetic mice by adenovirus-mediated glucokinase expression. Diabetes 50:2287-2295

11. O'Doherty RM, Lehman DL, Telemaque-Potts S, Newgard CB (1999) Metabolic impact of glucokinase overexpression in liver. Lowering of blood glucose in fed rats is accompanied by hyperlipidemia. Diabetes 48:20222027

12. Belfiore F, Romeo F, Iannello S, Salamone C (1989) The glucose-6-phosphatase/glucokinase ratio in the liver of obese-diabetic subjects. Biochem Med Metab Biol 41:7780

13. Huuponen R, Karvonen I, Sotaniemi E (1989) Activity of hepatic glucose phosphorylating and NADPH generating enzymes in Zucker rat. Diabetes Res 10:143-146

14. Davidson AL, Arion WJ (1987) Factors underlying significant underestimations of glucokinase activity in crude liver extracts: physiological implications of higher cellular activity. Arch Biochem Biophys 253:156-167

15. Keppler D, Decker K (1981) Glycogen. In: Bergmeyer HU (ed. in chief) Methods of enzymatic analisis, vol VI. Verlag Chimie, Weinheim, Germany, pp 11-18

16. Michal, G. Glucose 6-Phosphate (1981) In: Bergmeyer HU (ed. in chief) Methods of enzymatic analisis, vol VI. Verlag Chimie, Weinheim, Germany, pp 185-190

17. Carr TP, Andresen CJ, Rudel LL (1993) Enzymatic determination of triglyceride, free cholesterol, and total cholesterol in tissue lipid extracts. Clin Biochem 26:39-42
18. Niswender KD, Postic C, Jetton TL et al. (1997) Cellspecific expression and regulation of a glucokinase gene locus transgene. J Biol Chem 272:22564-22569

19. Stumvoll M, Jacob S (1999) Multiple sites of insulin resistance: muscle, liver and adipose tissue. Exp Clin Endocrinol Diabetes 107:107-110

20. Unger RH (1995) Lipotoxicity in the pathogenesis of obesity-dependent NIDDM. Genetic and clinical implications. Diabetes 44:863-870

21. Lewis GF, Carpenteir A, Adeli K, Giacca A (2002) Disordered fat storage and mobilization in the pathogenesis of insulin resistance and type 2 diabetes. Endocr Rev 23:201229

22. McGarry JD (2002) Dysregulation of fatty acid metabolism in the etiology of type 2 diabetes. Diabetes 51:7-18

23. Prentki M, Corkey BE (1996) Are the beta-cell signaling molecules malonyl-CoA and cytosolic long-chain acylCoA implicated in multiple tissue defects of obesity and NIDDM? Diabetes 45:273-283

24. Goto T, Onuma T, Takebe K, Kral JG (1995) The influence of fatty liver on insulin clearance and insulin resistance in non-diabetic Japanese subjects. Int J Obes Relat Metab Disord 12:841-845

25. Kusunoki M, Tsutsumi K, Hara T et al. (2002) Correlation between lipid and glycogen contents in liver and insulin resistance in high-fat-fed rats treated with the lipoprotein lipase activator NO-1886. Metabolism 51:792-795

26. Seppala-Lindroos A, Vehkavaara S, Hakkinen AM et al. (2002) Fat accumulation in the liver is associated with defects in insulin suppression of glucose production and serum free fatty acids independent of obesity in normal men. J Clin Endocrinol Metab 87:3023-2028

27. Seip M, Trygstad O (1996) Generalized lipodystrophy, congenital and acquired (lipoatrophy). Acta Paediatr Scand Suppl 413:2-28

28. Moitra J, Mason M, Olive M et al. (1998) Life without fat: a transgenic mouse. Genes Dev 12:3168-3181

29. Gavrilova O, Marcus-Samuels B, Graham D et al. (2000) Surgical implantation of adipose tissue reverses diabetes in lipoatrophic mice. J Clin Invest 105:271-278

30. Bruning JC, Michael MD, Winnay JN et al. (1998) A muscle-specific insulin receptor knockout exhibits features of the metabolic syndrome of NIDDM without altering glucose tolerance. Mol Cell 2:559-569

31. Michael MD, Kulkarni RN, Postic C et al. (2000) Loss of insulin signaling in hepatocytes leads to severe insulin resistance and progressive hepatic dysfunction. Mol Cell 6:87-97

32. Valera A, Pujol A, Pelegrin M, Bosch F (1994) Transgenic mice overexpressing phosphoenolpyruvate carboxykinase develop non-insulin-dependent diabetes mellitus. Proc Natl Acad Sci USA 91:9151-9154

33. Rosella G, Zajac JD, Baker L et al. (1995) Impaired glucose tolerance and increased weight gain in transgenic rats overexpressing a non-insulin-responsive phosphoenolpyruvate carboxykinase gene. Mol Endocrinol 9:1396-1404

34. Thorburn AW, Baldwin ME, Rosella G et al. (1999) Features of syndrome $\mathrm{X}$ develop in transgenic rats expressing a non-insulin responsive phosphoenolpyruvate carboxykinase gene. Diabetologia 42:419-426

35. Sun Y, Liu S, Ferguson S, Wang L et al. (2002) Phosphoenolpyruvate carboxykinase overexpression selectively attenuates insulin signaling and hepatic insulin sensitivity in transgenic mice. J Biol Chem 277:23301-23307 